\title{
The Exploration of New Multidimensional Reading Methode
}

\author{
Zhang Yuan \\ Master of Art and Design, Tsinghua University, Beijing, China
}

Email address:

15210086196y@163.com

\section{To cite this article:}

Zhang Yuan. The Exploration of New Multidimensional Reading Methode. Science Innovation. Vol. 9, No. 4, 2021, pp. 156-163. doi: $10.11648 /$ j.si.20210904.17

Received: April 20, 2021; Accepted: June 17, 2021; Published: June 22, 2021

\begin{abstract}
Through a special combination of the full-text information and the graphic vision to design a valuable way of reading mode for electronic books. With the progress of current society, the people's reading modes and the medium of reading have been changed gradually at the some time, which brings a series of changes in the way of reading modes. Therefore, It is particularly important and necessary for people to seek a new way of reading, which help people to recognize quickly the content of text, the structures of text, and the internal regulation of the text. This paper expounds the necessity of optimizing the visual and the multi-dimensional way mods, And then, the paper discusses several advantages of the multi-dimensional reading modes step by step. This new way of reading way can help readers to recognize immediately the structure and logic of all kinds of books, which can help to improve efficiently the reading speed. It turns two dimensional text into three dimensional text. Through three-dimensional images, people can recognize quickly the internal relationship of complex texts, such as the plot development of the novel, the changes of different geographical information positions, and the different system and network of the literature knowledge.
\end{abstract}

Keywords: Reading Methodology, Graphical, Introduction, Reading Efficiency

\section{多维阅读方式的探索}

\section{张垣}

清华大学美院视觉传达系, 北京, 中国

\section{邮箱}

15210086196y@163.com

摘要：通过文本信息和图形视觉相结合设计一种有价值的电子书籍的阅读方式。随着社会的进步，人们阅读方式和文 本载体的变化, 带来的是阅读方式的变化。寻求一种供人们快速认知文本内容、结构和内在规律的阅读方式就显得尤 为重要了。文中阐述了优化阅读方式的视觉化和多维化的必要性, 然后一步步探讨了多维阅读方式的优点。这种阅读 方式能够帮助快速认知书籍的结构逻辑, 提高阅读效率。它将二维文本变成了立体文本。使人们通过立体图像快速认 知复杂文本的内在关系: 如小说的情节发展, 地理信息位置变化, 文献的知识的体系网等。

关键词：新型阅读方式, 图形, 导读, 阅读效率 


\section{1. 引言}

\section{1. 阅读方式现状分析}

电子图书的普及给传统纸质图书到来了很大的挑战 与冲击, 纸质图书与电子图书在未来的发展已引起广泛的 关注[1],2017年4月公布的《第十四次全国国民阅读调查报 告统计》的数据颠覆了很多国人的认知, 电子文本的阅读 率已达到 79.9\%, 见图 1、图 2。

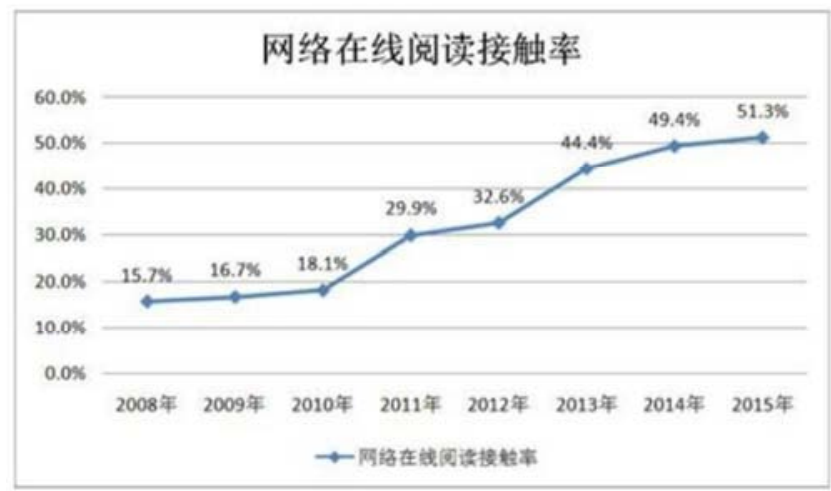

图1《阅读调查报告统计》。

手机阅读接触率

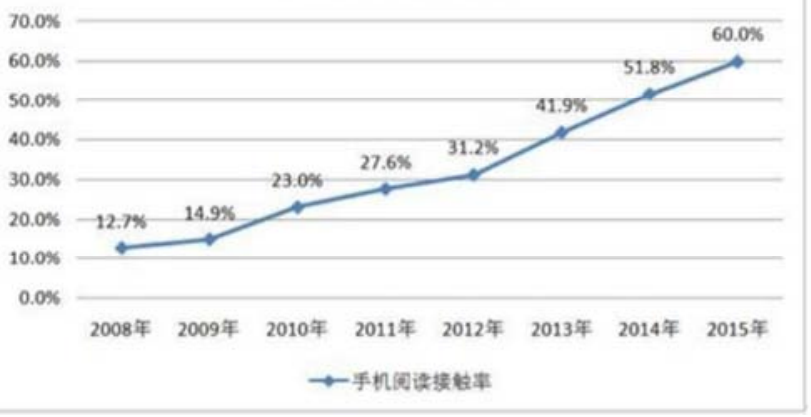

图2《阅读调查报告统计》。

\section{2. 阅读诉求现状分析}

在社会竞争日益激烈下,人们开始不断的提高自身的 素养和完善知识存储量。实用性工具书的阅读、跨专业阅 读、可操作性阅读、交互互动式读书等的高效率的阅读成 为了目前的趋势, 阅读面变得更加的宽泛和跨学科, 从成功 学类、励志类、营销类、外语类书、财经理财类,心理学 等, 阅读需求的变化驱动着阅读方式的变化[2]。1.3 目的 与意义

\subsection{1. 目的}

本研究的目的是在电子阅读的的基础上充分发挥视 觉图形的优势,通过文

本和图形语言的结合, 来设计一种新型的电子图书阅 读方式。本研究从提高效率的角度出发, 将电子书从二维 文本变成了可视化、立体的文本, 通过立体文本快速认知 文本的内在关系 1 。
1.2.2. 意义

本研究最大的意义在于提出了新型的阅读的形式, 一 方面本研究能直接获取该电子书籍的“文本信息源”2“重 点知识”起到导读-引导阅读的作用。通过它我们能够与书 籍发生互动,利用多维度 3 的文本模型进一步的查看和检 索关键词, 以一种阅读者想获取的对象为出发点的角度进 行阅读,提高阅读效率,

另一方面,本研究在原文本之内进行转化, 根据内容的 关联性构成新结构关系（三维图）：如小说文本中故事的 情节发展, 文献文本中的知识体系网等。带给读者完全不 一样的多维度阅读体验。

1 比如文中的知识点之间的关系、情节发展的关系。 通过查看立体文本, 迅速获取所需知识点。

2 文本中 作者最想传达的信息, 如知识点、若干条 建议等。

3 该模型在阅读时, 可以在空间上从任意角度进行跳 读, 打破了逐字逐句的线性阅读。

\section{2. 视觉语言的在阅读中的应用}

\section{1. 视觉的的重要性}

目前的阅读中,除了在文本中加入的图片外(见图 3、 图 4), 文本本身过于平白直述, 呈现的形态单一、乏味。如 今随着人们阅读方式的多元化, 文本的也需要具有视觉性、 审美性的设计[3], 这样才能满足随之提高个人审美的诉求。

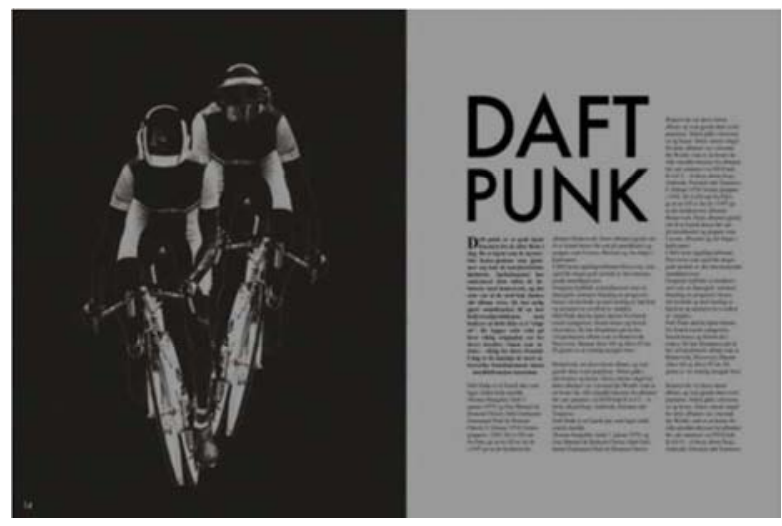

图3 排版案例。

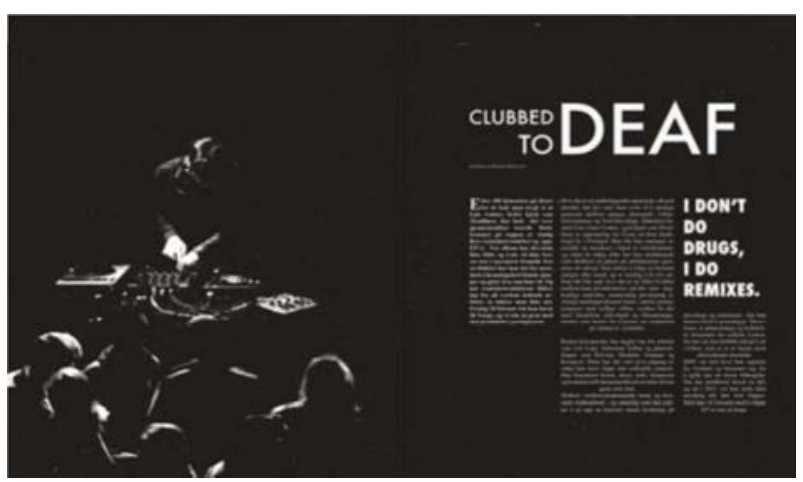

图4 组合案例。 


\section{2. 电子文本的视觉语言}

在文本中视觉语言编排甚至可以弥补在原文叙述上 的欠缺, 以及非线性阅读的缺陷[4]。它通过人对于外部信 息的感受与体验获取, 再以视觉语言的表演形式传达给 读者, 是一种视觉化的高效传递方式[5]。在电子文本中, 语 言文字的构成是抽象化和图形化的视觉系统。文本的图像 化传达了设计师的设计理念和文本的内涵,这些构成一种 可阅读、可视化的综合型文本(电子书籍)形态。

本研究是图像与视觉语言的结合体。在文本的导读部 分中可以按照如小说文本中主人公的感情线路发展为视 觉语言, 以《罗密欧与朱丽叶》海报 $\mathrm{a}$ 为例, 海报红色部 分就是两位主人公的爱情历程的二维图 (见图 5、图 6), 除此之外还有历史类别的地理信息位置的变化,文献文本 中的知识的体系网等。本研究实在在三维空间中重新编排, 让文本本身形态丰富、充满趣味性。

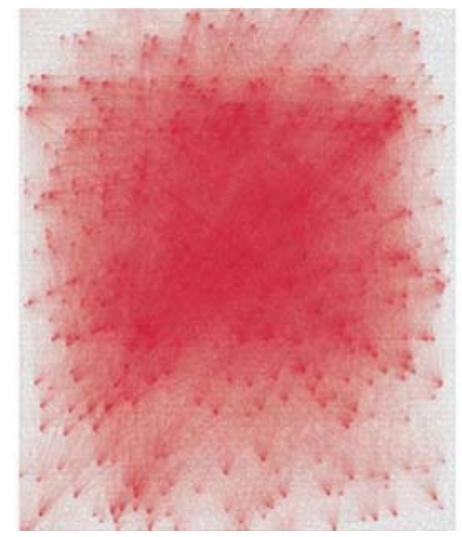

图5 海报。

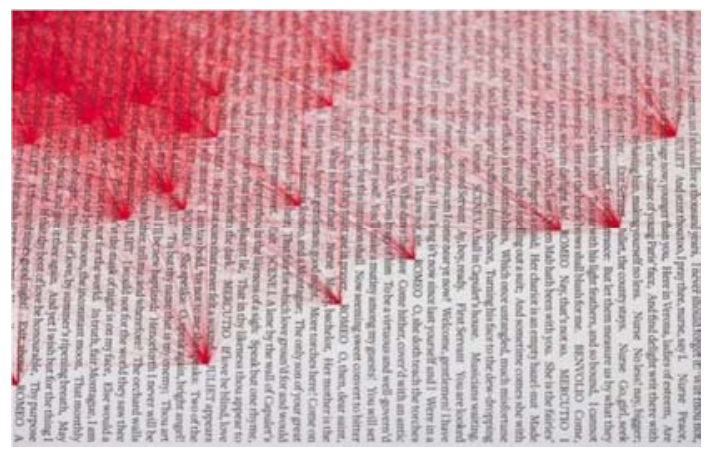

图6 单词。

\section{3. 阅读方式的多元与多维}

论述的“多维性”是指两个方面，一方面“多维性”是指 时代的不同,阅读方式的多元化。阅读方式革新也是为了 满足文本载体的不断演变。线性和非线性、浅阅读和深阅 读、跳读和网状阅读等阅读方式交错出现 [6]。将传统的文 本转化为视觉文本和电子文本, 在枯燥的线性阅读中增加 图像符号和互动性体验。阅读不会消亡, 只是随着。

$\mathrm{a}$ 该海报获得红点设计的海报奖，它基于文本分析和 文本可视化的基础上设计的。 $\mathrm{b}$ 在阅读行为上, 不需要逐行阅读, 可以按读者的意 愿图任意角度去抓取词, 通过模型的阅读是三维的阅读行 为。

社会的不断变迁,载体和阅读方式都会不断革新, 比 如电子书 kindle 和听书app。见图 7、图 8。

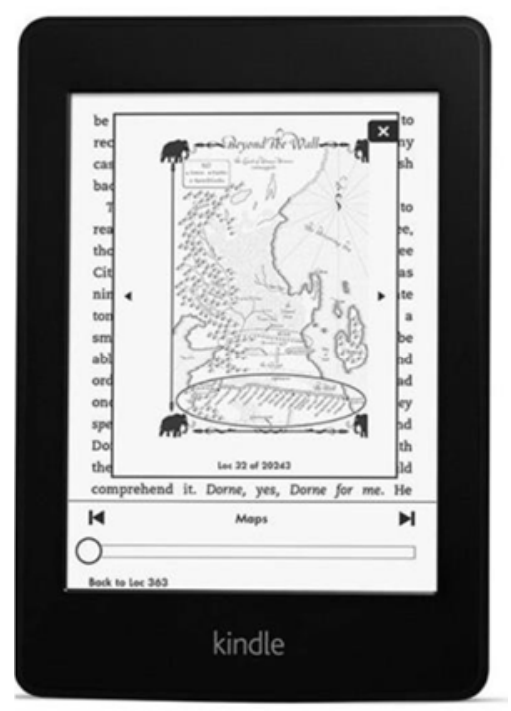

图7 kindle。

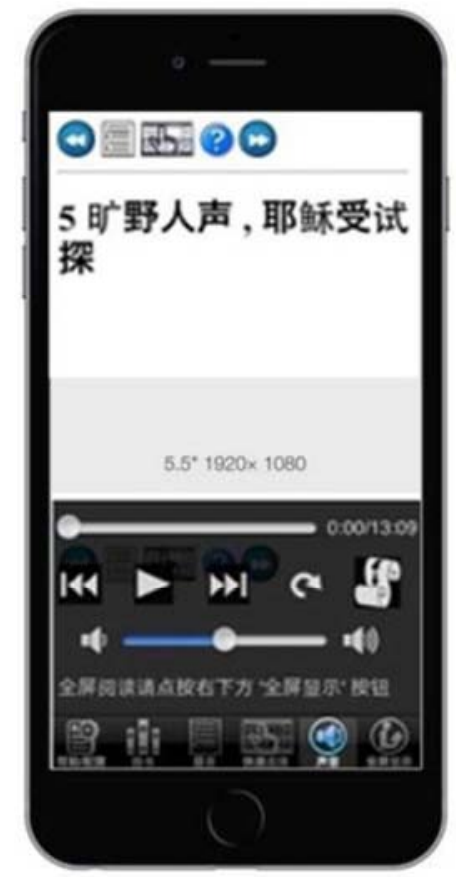

图8 听书app。

\section{4. 阅读行为的多维性}

另一方面“多维性”是2.1指文本空间上的位置分布。电 子书在空间上是平面的阅读、从结绳记事到文本记事, 阅 读的行为都是线性的、平面的阅读。信息的获得过程是由 局部到整体,由线到面,再由面及点（知识点）[7]。三维阅 读方式（见图 9、图 10）在空间上是立体的阅读,可以 按读者的意愿图从任意角度去抓取词, 是三维的、跳跃性 
的选择性阅读。人眼捕获信息时,是把整本书看作一个整 体,由整体到局部、由面到点(知识点)的过程。

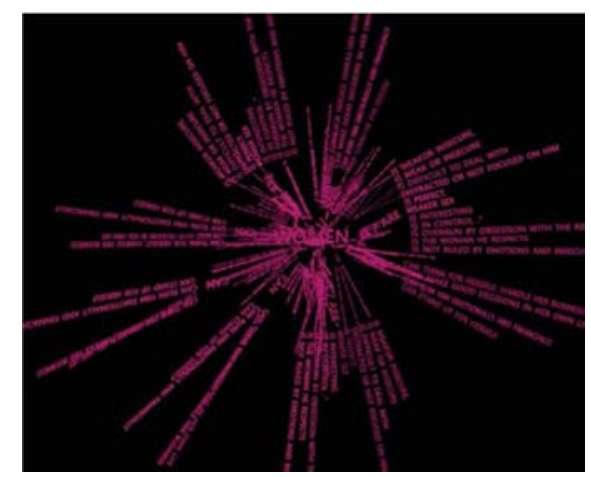

图9 文本脉络图。

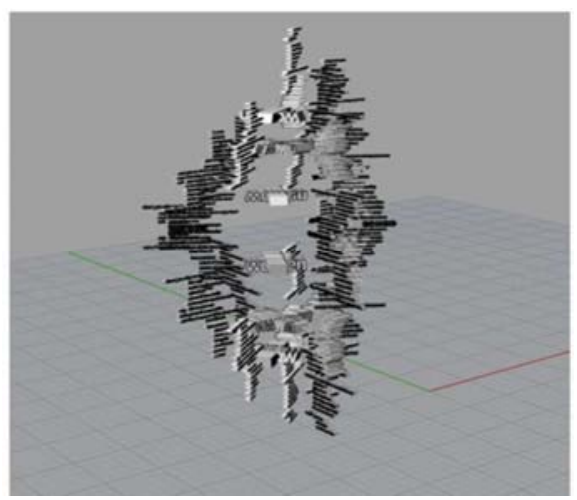

图10 词汇模型。

多维指的并不是图文并茂,而是基于自然语言处理技 术。自然语言处理技术 (NLP)包括命名实体识别、关系抽 取、文本分类等 $[8]$, 利用电子书籍的的数据化和文本视觉 化的特征, 方便读者能够利用视觉感知来快速的获取其中 所蕴含的重点知识(信息), 见图 11。

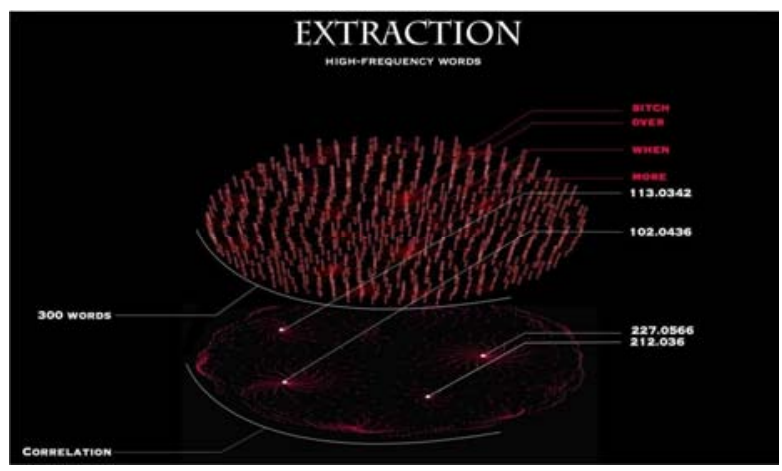

图11 单词提取。

\section{3. 实践与探索}

\section{1. 概念解析}

多维度阅读方式能够将文本以三维图形的形式展示 出来, 读者每本文本一个直观的了解, 从而起到一个导读的 作用。其次, 它符合当下的碎片化阅读阅读趋势 [9], 让读
者在文本中跳跃性的选读所需知识点,尤其在阅读大量文 献时显得尤为效率。最后它能够通过检索相关关键词在 文本中畅读。

\section{2. 实践前期}

\subsection{1. 创作初衷}

在使用网电子籍的网站汶览书籍时, 笔者发现并不能 直观的进一步判断和了解这本电子书的内容 (见图 12) 所以在文本可视化的基础上尝试设计了一种新的阅读方 式, 对比见图 13:

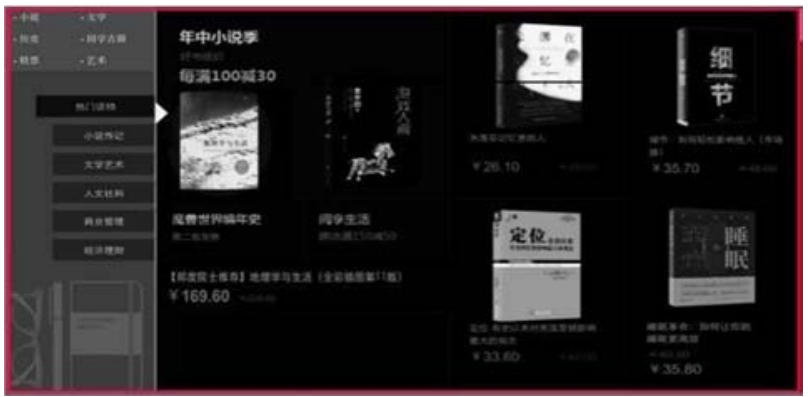

图12 某网上书店。

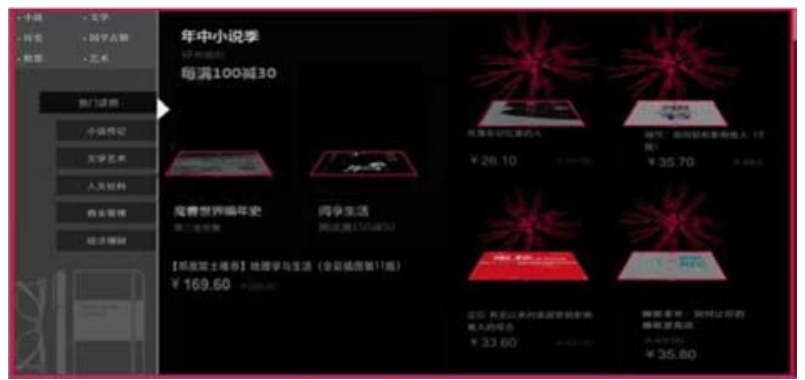

图13 概念网上书店。

\subsection{2. 创作对象}

现场选取了三本不同的文本为案例展示 (见图 14、 图 15、图 16）,由于文本的内容之间有着密切的联系: 如历史类别的地理信息位置的变化,文献文本中的知识的 体系网等。将其分析、提取、再深化加工后设计出合乎

其联系与内容的表现图形。如果仅仅提取的是局部内 容展示,便无法起到导读全文的作用了,所以本研究的范围 是整个文本。读者可以在开启导读阅读模式时纵览全局, 利用人与生具来的视觉感知获取关键信息。见图 17

\section{案例1:}

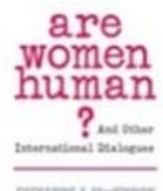

图14 文本案例。 


\section{案例2:}

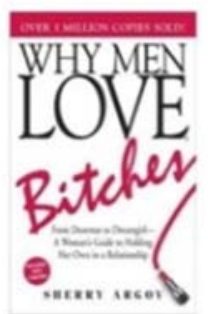

图15 文本案例。

案例3:

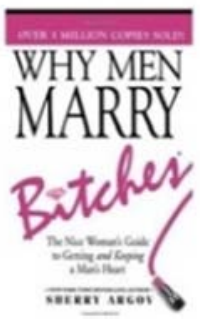

图16 文本案例。

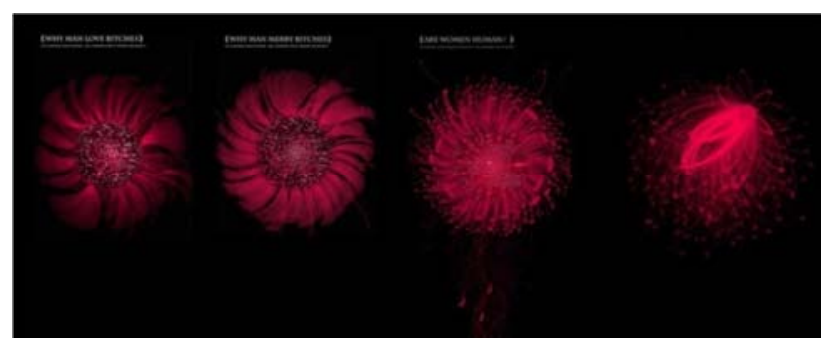

图17 数据生成图。

\section{3. 具体实践}

多维阅读方式的呈现关键在于文本三维化、文本视觉 化。所以在此之前需经历 4 个步骤:

\subsection{1. 提取所需文本}

分析文本中每个词出现的频率和与它相关内容的如 图 18 图 19

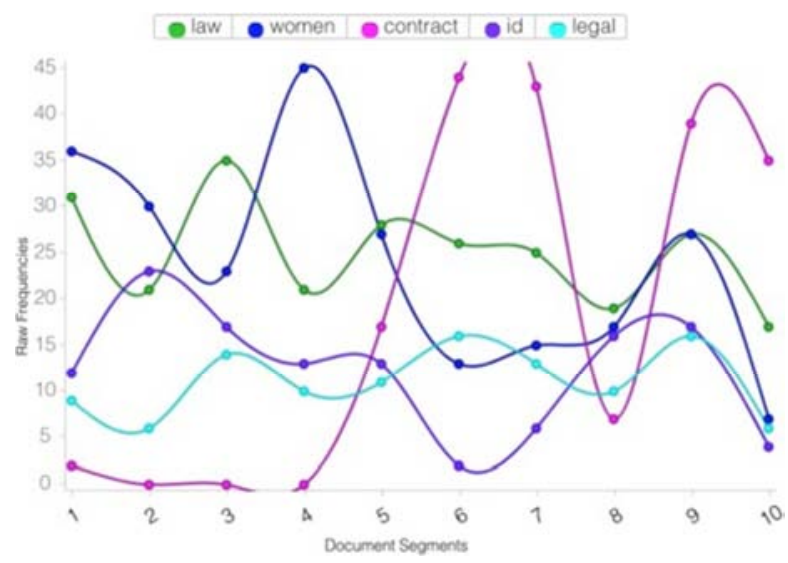

图18 词频统计。

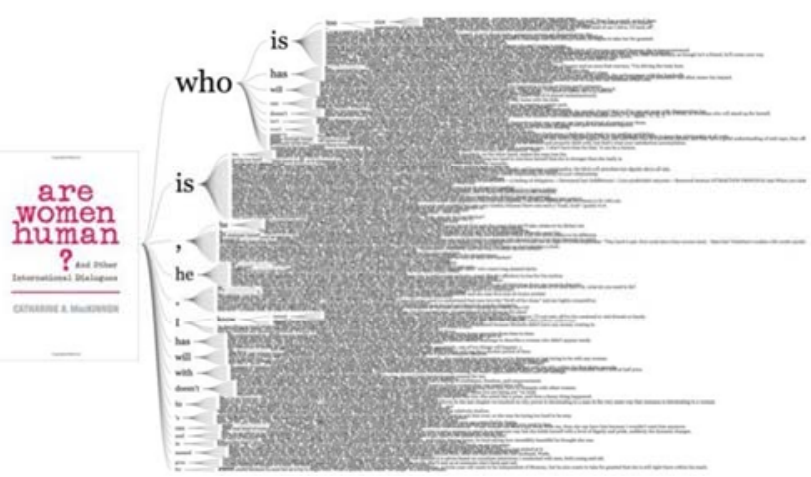

图19 词发散图。

\subsection{2. 分析文本}

包含事件、时间、关系、等二位的脉络图(二维图形) 如图 20、图 21、图 22、图 23、图 24 .

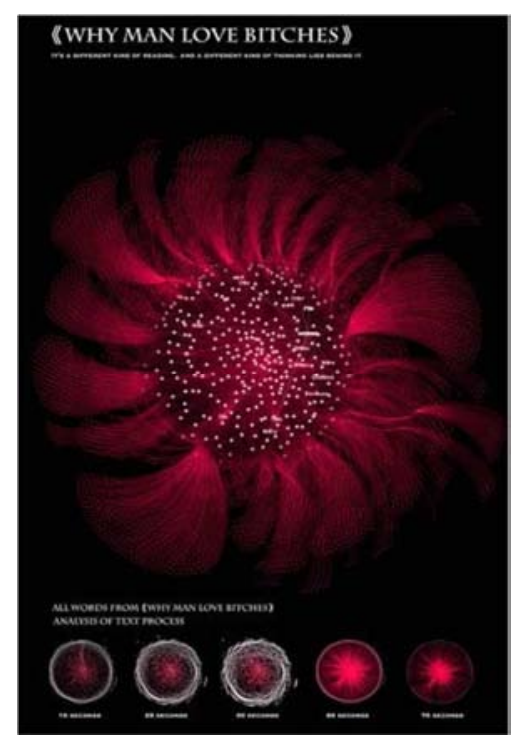

图20 文本案例一。

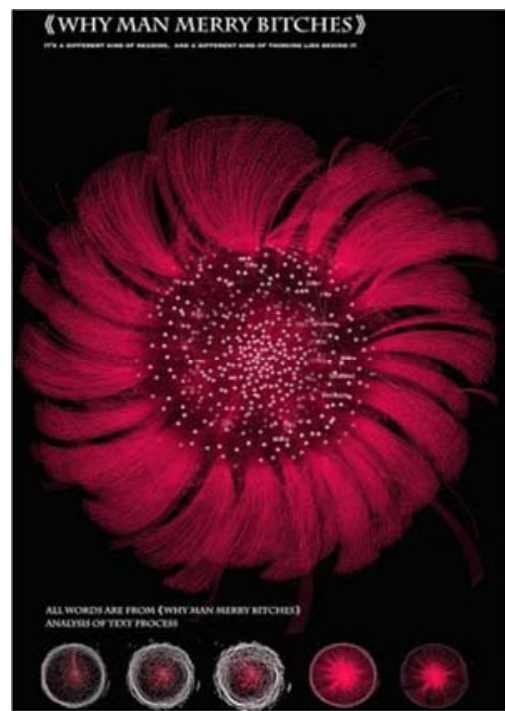

图21 文本案例二。 


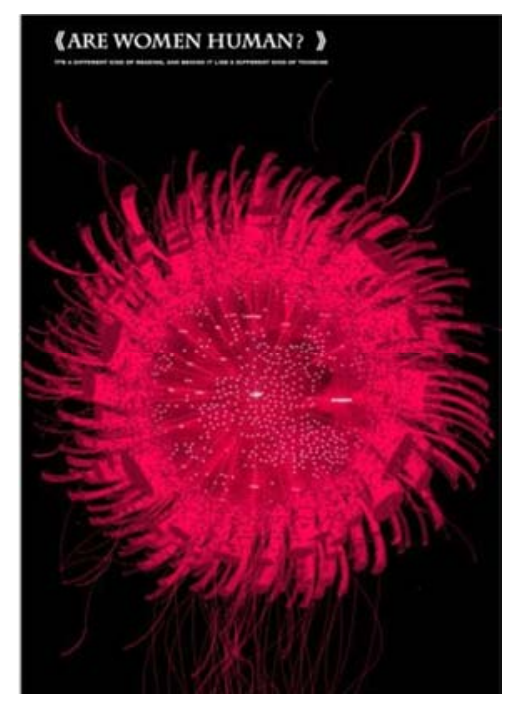

图22 文本案例三。

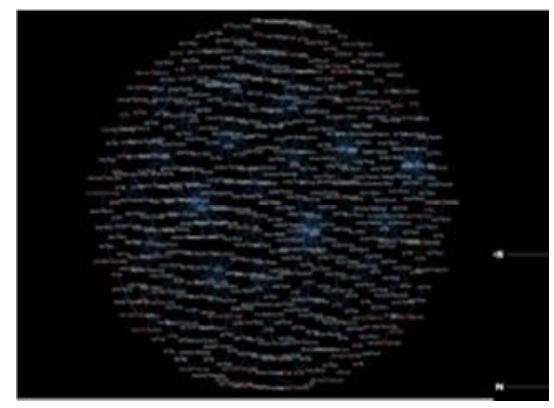

图23 提取后二维图。

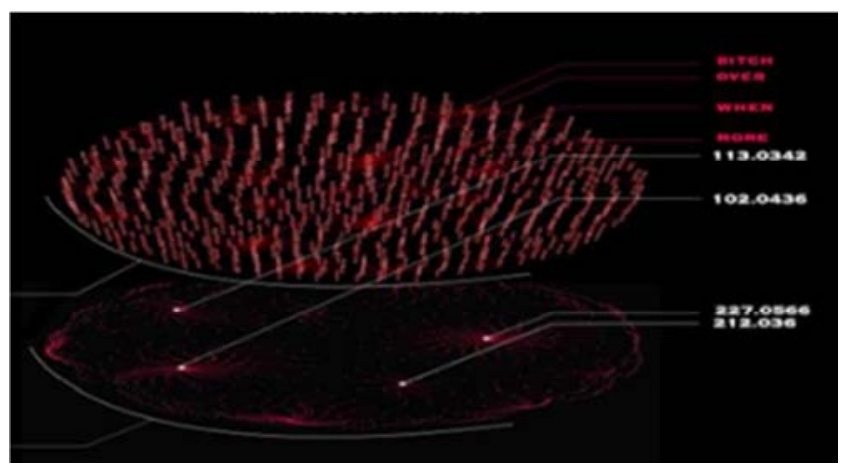

图24 二维图结构。

在文本分析中,包含词语、语法和语义三个等级[12]， 通常文本中关键词分析、文本分类别、是相对常用的技术。 情感强烈的文本进行分析和架构的过程, 以作品 《why man marry bitches?》为例,该文中结合一些事例将 78 条原则呈 现给读者。通过笔者设计的三维阅读方式,则可以统揽全 文,迅速的获取文中 78 条核心建议。以上需要首先分析 《why man marry bithes?》的文本内容,提取所需内容。再 分类它的文本性质、统计文本的词汇和内容的相关性。根 据分析, 在图二中通过文本中的高频频率出现的关键词可 以明显的看出《why》系列是围绕男女关系, 以忠告女性在 男女相处之间注意事项。原则建议为主, 所以提取出来的 内容围绕“principle”一词。

\subsection{3. 视觉化呈现-生成三维模型, 见图 25}

在三维文本中 [10],三维文本类似于树枝发散的形状, 在此步骤中的最为重要的就是选择一个合适的视觉语言 (编码),例如词频的高低决定了字的大小,情感色彩的倾向 决定了最后三维文本的颜色。例如在《why》系列,词频出 现最高的是“man”“woman”三个词,因为它是围绕男女关系 展开的论述,所以在最终的三维图像中这两个高频词的字 体也就显得格外的大。而在在讲述法律与女权的《are woman human?》中的最终三维图像中,“law”在最终图形 中呈现的字体较大, 因为它是这本书籍的高频词之一。另 外在进行检索时,将会出现输入款,便于读者输入想检索的 文本信息。

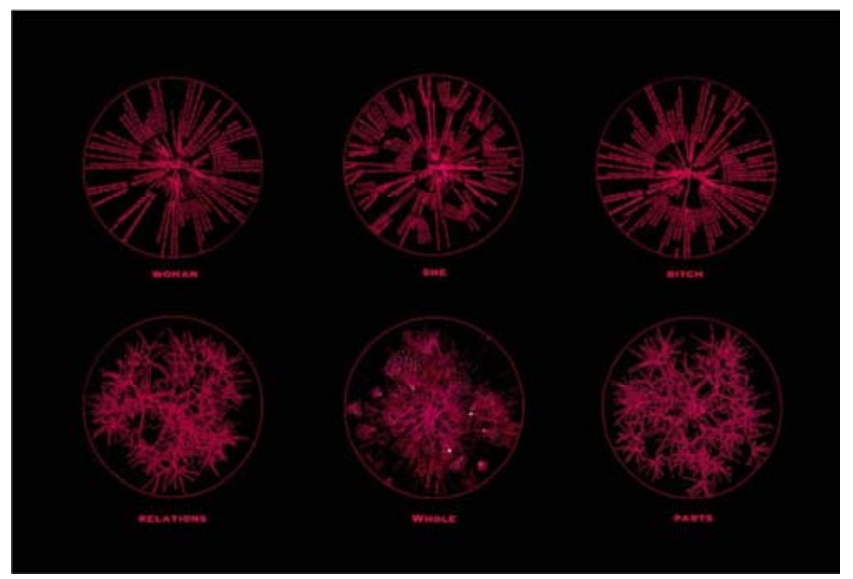

图25. 局部建模。

\subsection{4. 完成用户与文本的互动组成, 如图 26}

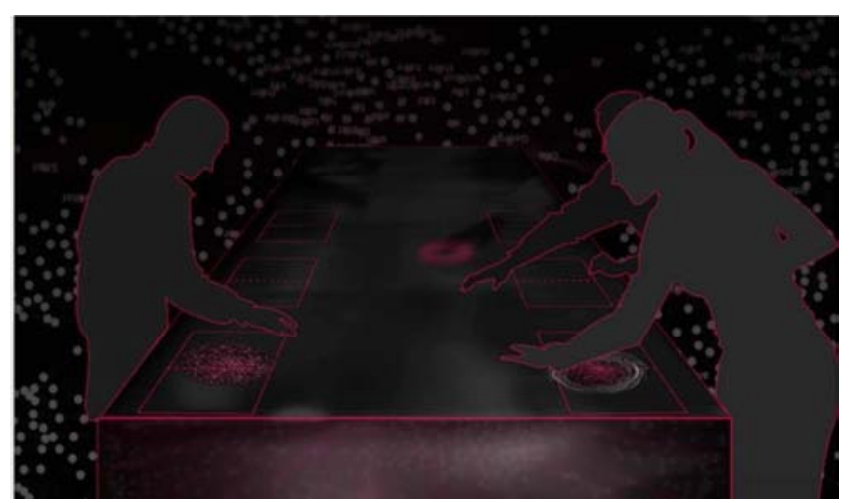

图26 应用场景前景。

\section{4. 研究结果}

\section{1. 文本的三维性}

本研究将电子文本以多维化后, 具有以下三类特征。 不同于首先是文本的三维性[11], 这是一本与众不同的立 体电子书按照提取内容的形式划分图形空间的分布,按照 关键词与内容相关性来扩展或检索的它本身(发散性), 见 图 27、图28。 


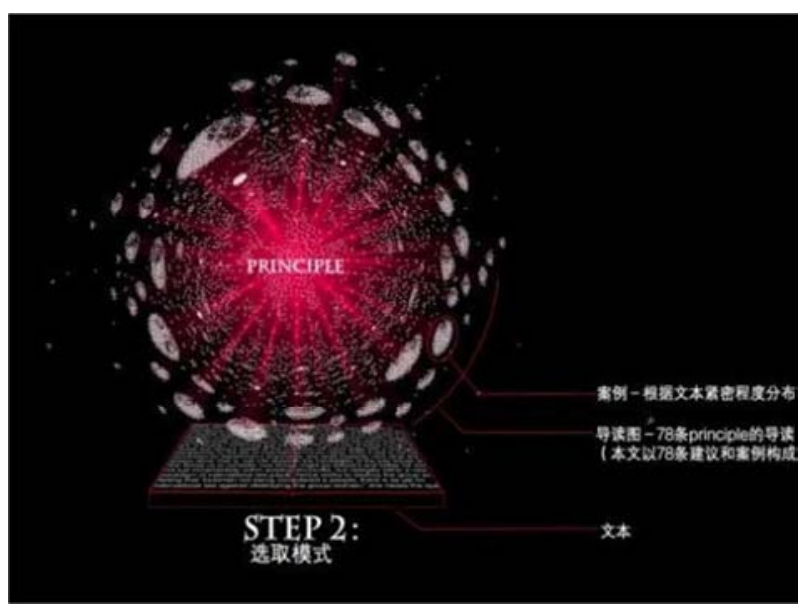

图27 模拟。

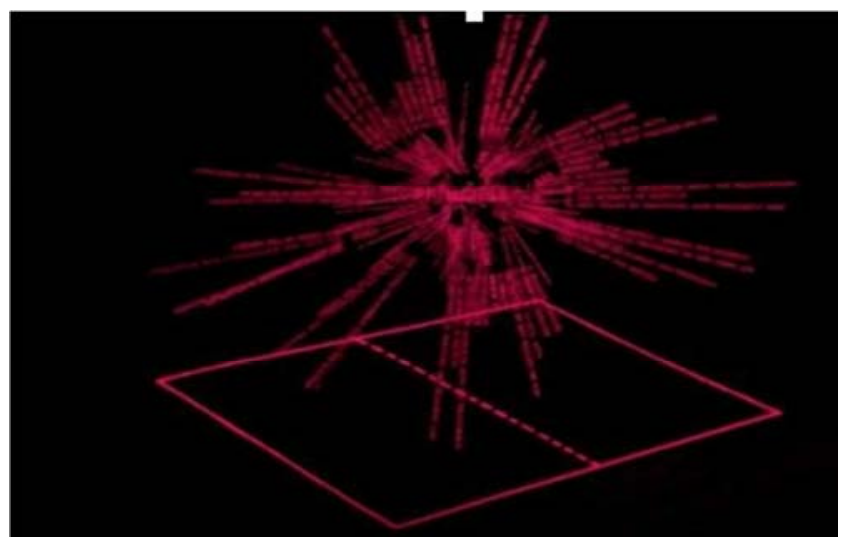

图28 建模模拟。

经过统计、提取、整合文本后,参考大脑神经元的形 象,将文本用三维图形的方式展示出来，不同于一般书籍 以平铺直叙的文字呈现, 它可以在空间中进行内容的比较 阅读,甚至是检索更多的内容,所以它是从整体到局部(关 键词), 再通过提取关键词和基于关键词权重检索 [13],由点 至面的,构造一种新的阅读行为,让书不仅仅是作为载体的 单向信息传达,而是呈现一种可以反相(双向)的探索书中 谋一个点, 从而形成一种有点至网的三维阅读体验。

\section{2. 导读功能}

传统的阅读方式是按照作者的逻辑,文本的线性走向, 由浅至深的阅读文章, 数字化的阅读是近年来的趋势, 与 纸质书相比, 讨论谁取代或存亡的问题并没有太大的价值, 而是把各自处于最能发挥自身优势的领域。

而大多数情况“前沿介绍”过于片面,同时难以包含读 者寻找的知识点/关键词。所以不讨论纸质文本或者电子 文本, 而是运用现有的优势, 提供更多的可能性,是一种文 本(书籍)概念设计,由于它承载的内容是统计、提取、整合 文本后的, 它能够在读者阅读这本书之间,通过阅读整个三 维图形,对于读者起到一个导读的作用, 并且分文本的层次, 同时能够进行某个知识点的检索, 具体操作见图 29、图 30、图 31:

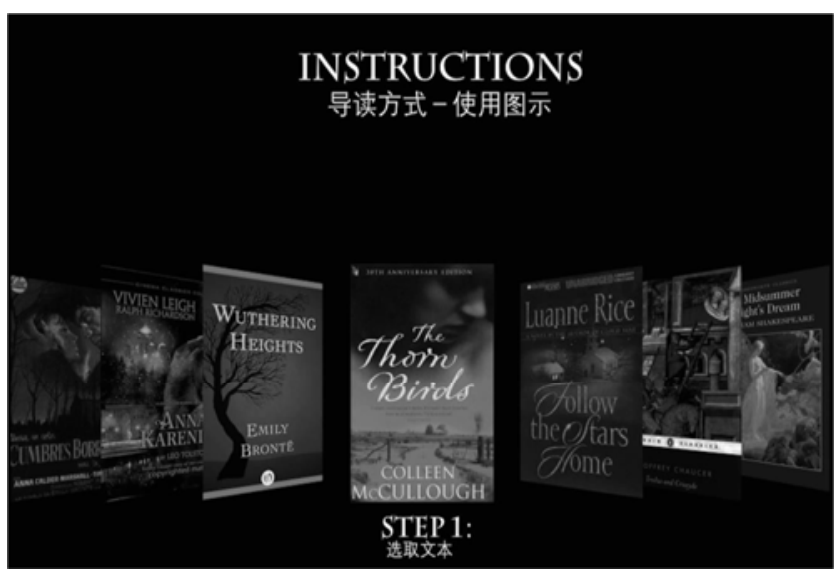

图29 步骤一图示。

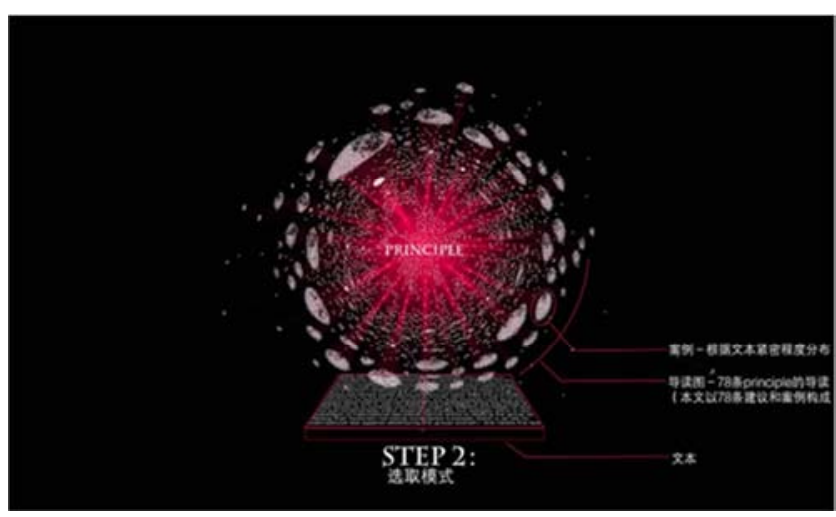

图30 步骤二图示。

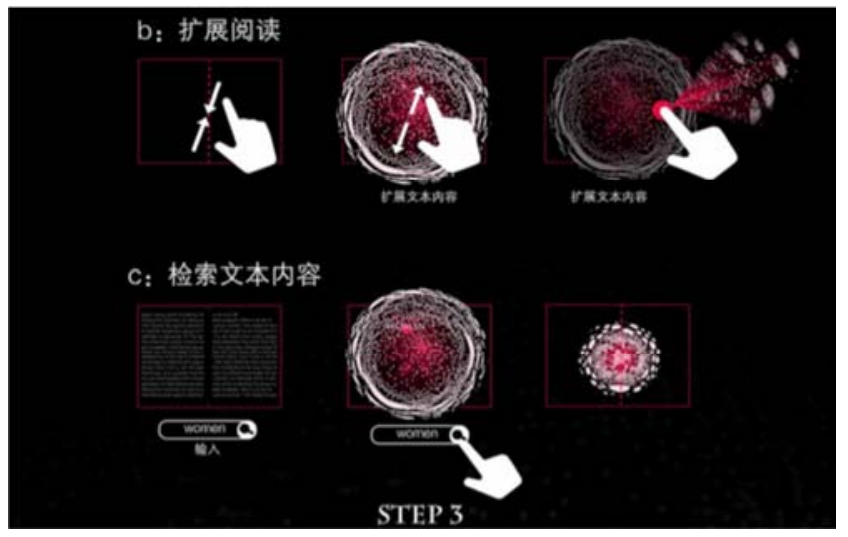

图31 步骤三图示。

\section{3. 互动的阅读体验}

非线性阅读不同于线性阅读,它根据文本关系,不断的 出现新的连接节点或者关系连线。构成新的网络。可以进 行非线性的扩展阅读、发散性阅读。而且这种互动的阅读 方向可以实现阅读时思维的自由。

多维度阅读方式的互动行为分三层,第一个层次来选 定喜爱的区域,第二层可以进一步的检索这个区域相关的 文本由于成个三维文本的架构是根据不同内容的不同特 性进行结构分配的,所以在最后呈现的文本中,会出现于区 别于原本文本内容,在空间上呈现出不同的区域见图 32、 图 33。 


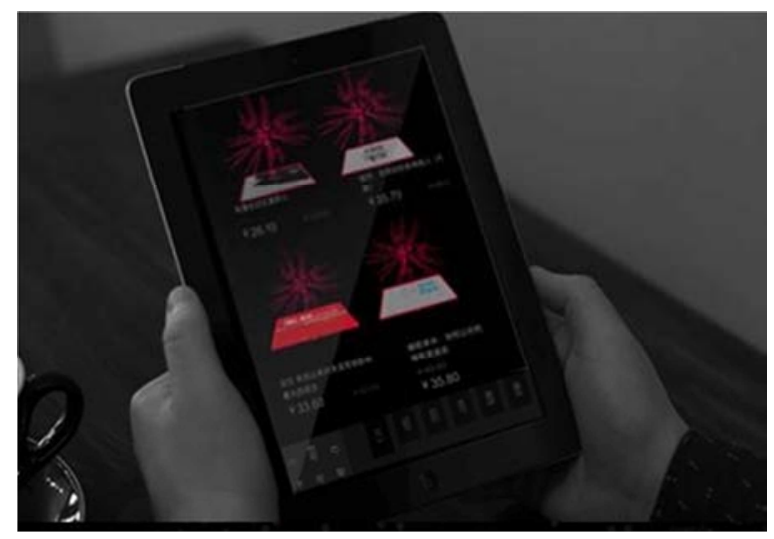

图32 应用前景。

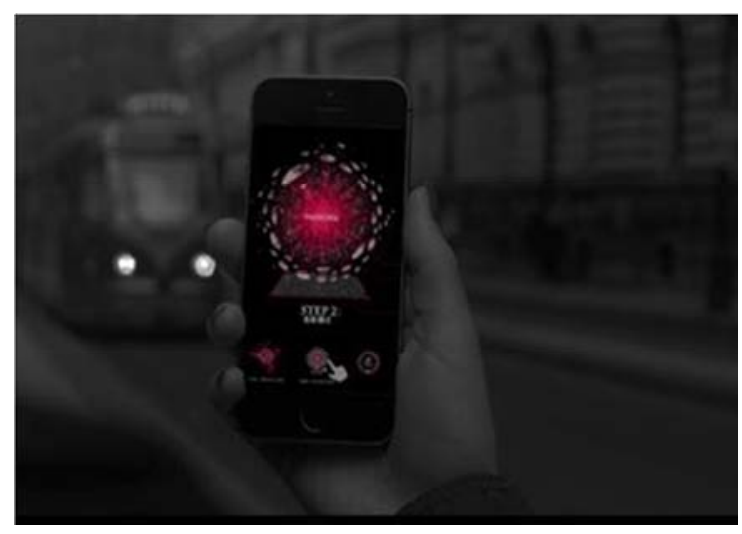

图33 移动媒体。

\section{5. 结论}

基于文本可视化的的多维阅读方式具有维度性、互动 性、不同媒介应用性等优势, 也是未来阅读电子文本的 一个发展方向。电子文本的结构通过多维度化的呈现, 赋 予了读者阅读电子文本一个不同的视角, 使得每本电子书 籍拥有自己独一无二的特性与视觉特征。并且在碎片化阅 读趋势的时代背景下, 这种创新型的阅读方式能够帮助读 者快速认知书籍的结构逻辑, 提高阅读效率, 帮助读者快 速认知复杂文本的内在关系: 如小说的情节发展, 地理信 息位置变化, 文献的知识的体系网等。多维阅读方式凭借 着其直观的视觉化呈现，为不同领域的人提高了更效率的 阅读体验, 并且由于其应用载体十分广, 有一定的市场前 景。

多维阅读方式作为一种新型的阅读探索, 也面临着一 个需要解决的问题——如何协调好文本转化后的模型与 读者阅读体验的关系。所以在下一步的研究上, 主要在研 究的重点是将进一步完善读者在使用多维阅读方式的的
体验感, 不同类别文本的阅读, 追根溯源都有着其文本自 己的结构, 所以多维阅读方式并不是独立存在的一种阅读 手段, 而是基于文本本身词频、语义、结构等多个内容集 成的一种文本的转换手段。因此, 在转化过程中的所有的 可视化、数据化技术都是服务于人, 以人为本才。以读者 在阅读时的使用体验为出发点是新型阅读方式能够再进 一步发展的安身立命之本。

\section{参考文献}

[1] 张凤叶. 谈电子图书在阅读教学中的应用策略 [J]. 中华活页 文选(教师版),2020(03):80-82。

[2] 刘洪梅. 阅读变迁及对数字化阅读的思考 [J]. 中国传媒科技, 2012。

[3] 叶浩鹏. 叶浩鹏.现代书籍设计中的视觉语言表达[J].大众 文艺,2014(08):61-62。

[4] 曹方. 数字时代与非线性阅读.上海信息化 [J]. 2013 (4) : $82-84$ 。

[5] 李慧. 论视觉语言在书籍装帧设计中的运用[J].美与时代， 2010。

[6] 焚晓春.多元化阅读模式对大学图书馆信息资源建设的影 响[M].云南:云南图书馆 2010。

[7] 王凤英.语文阅读的教学对话: 从线性思维走向非线性思维 [J].福建基础教育研究,2019(07):35-37。

[8] 张敏,丁良萍,刘欢.面向科技文献的多维语义索引构建思路 及 实现 $[\mathrm{J} / \mathrm{OL}]$. 情 报 理 论与 实 践 :1-10[2021-05-11].http://kns.cnki.net/kcms/detail/11.1762. G3.20210413.1752.009.html。

[9] 马志红. 碎片化阅读时代网络媒体深度报道问题及策略研 究 [J].新媒体研究,2018,4(04):76-77.

[10] 顾鸿. 语文教学中的线性阅读与网状阅读.[J]. 语文天地， 2013。

[11] 周磊, 刘芳芳.XYZ三维阅读法构建个性知识网络[J].科技视 界,2020(29):1-3。

[12] 朱巧.基于词频统计的文本可视化实现 [J].软件,2016，37 (11) :114-117。

[13] 林跃.基于语义的文本可视化研究.[D]哈尔滨工程大学, 2014。 